\title{
TOOLS TO PREDICT BEAM BREAKUP IN RECIRCULATING LINACS*
}

\author{
K.Beard, L.Merminga, B.Yunn, TJNAF, Newport News, VA 23606, USA
}

\begin{abstract}
An important limitation on the maximum beam current in a recirculating linac is due to beam breakup caused by higher order modes (HOM) excited in the RF cavities. A HOM delivers a transverse kick to a beam bunch, the bunch on the next pass can then drive the HOM and cause it to grow until the beam is lost. Two codes, MATBBU and TDBBU, have been written to estimate the threshold current for a set of HOMs and accelerator optics. The relative merits and limitations of each is discussed in detail.
\end{abstract}

\section{INTRODUCTION}

Often the greatest beam current limitation in a recirculating linac is imposed by beam breakup (BBU) caused by transverse higher order modes (HOMs) in the accelerating cavities. The basic idea, very briefly, is that a bunch passing through a cavity off axis excites a transverse electromagnetic mode in the cavity. When the same bunch later returns to that same cavity, it will again interact with the same mode; under the correct circumstances, that mode will grow until it deflects the beam sufficiently to cause the beam to be lost. The exact combination of HOM characteristics, beam transport, and recirculation time determine whether a given mode will lead to an instability.

For a single recirculation, a singe cavity, and a single mode, the expression for the beam break threshold current is approximately: ${ }^{[1]}$

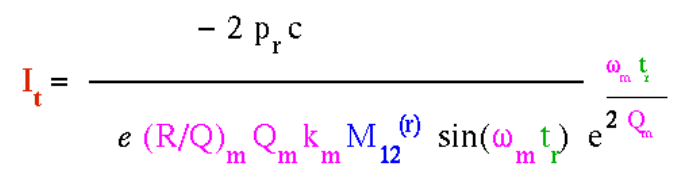

where $\mathrm{I}_{\mathrm{t}}$ is the threshold current, $\mathrm{p}_{r} \mathrm{c}$ is the momentum leaving the cavity, $e$ is the charge of the electron, $(\mathrm{R} / \mathrm{Q})_{\mathrm{m}}$ is the shunt impedance, $Q_{m}$ the quality factor of the mode, $\mathrm{k}_{\mathrm{m}}$ is the wavenumber of the mode, $\omega_{\mathrm{m}}$ is the frequency of the mode, $\mathrm{M}_{12}{ }^{(\mathrm{r})}$ is the component of the transport matrix, and $\mathrm{t}_{r}$ is the recirculation time.

\section{TDBBU}

Solving the problem for many modes in many cavities and multiple passes becomes more complicated. ${ }^{[2][3]}$ The JLab CEBAF $12 \mathrm{GeV}$ upgrade has a design current of 100uA and about $2600 \mathrm{HOMs}$ of interest; the Jlab $10 \mathrm{~kW}$ FEL has a design current of $10 \mathrm{~mA}$ and about 200 HOMs of interest.

One approach is to use the TDBBU code written by G.Krafft, modified by B.Yunn, and then by K.Beard. It works in the time domain as a tracking code. Individual bunches are injected into the machine; a small amount of noise is added, the bunches stepped through the machine, and each HOM mode updated. The process is repeated for a time long $(\sim 10 \mathrm{x})$ with respect to the growth time of a mode $\left(\sim 2 \mathrm{Q}_{\mathrm{m}} / \omega_{\mathrm{m}}\right)$, and the transverse positions of the bunches at a point in the machine are plotted. An instability appears as growth in the transverse position:
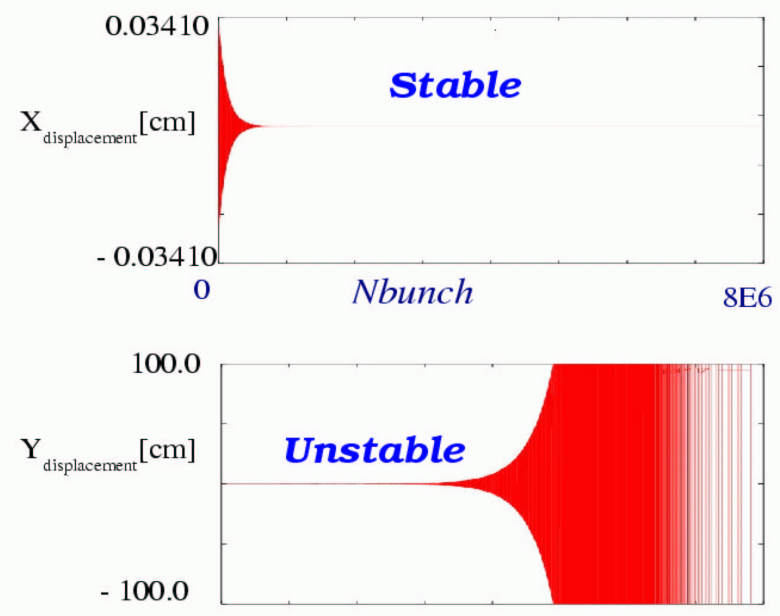

Figure 1: TDBBU output for stable $\left(\mathrm{I}<\mathrm{I}_{\mathrm{t}}\right)$ and unstable $\left(\mathrm{I}>\mathrm{I}_{\mathrm{t}}\right)$ examples.

To find the threshold current, the current used is turned up an down and the calculation repeated; an automatic algorithm to do this is included in the new version of TDBBU. It examines the output and decides whether the 
situation appears stable, unstable, or indeterminate, then adjusts the current appropriately and runs again.

As the required runtime goes $\sim \mathrm{N}_{\mathrm{HOMs}} * \mathrm{Q}_{\mathrm{m}} / \omega_{\mathrm{m}}$, this approach is becomes cumbersome for very high Qs. Another approach is to solve the problem in the frequency domain. If one assumes a steady state solution, the problem can be reduced to solving for the complex eigenvalues of a matrix. ${ }^{[6]}$

\section{MATBBU}

The MATBBU[7] code reads in the same input file as TDBBU. It then sweeps through a frequency range, for each frequency it calculates a matrix and searches for eigenvalues of the matrix. The lowest eigenvalue falling on the real positive axis correspond to the threshold current

In the initial case of the $10 \mathrm{~kW}$ FEL, there were 56 HOMs/axis, requiring that a complex $111 \times 111$ matrix's eigenvalues be found. This takes some significant time on any computer; in addition, the high Q's mean that the regions of interest are very narrow; only a few $\mathrm{Hz}$ wide. Typically, one would like to sweep a $2 \mathrm{MHz}$ wide space; doing that with just "brute force" by sweeping in $1 \mathrm{~Hz}$ steps requires $2 \times 10^{6}$ steps, or, very roughly for a $733 \mathrm{MHz}$ Pentium III, about that number of seconds (about 3 weeks) for each band (there were 11). In addition, this sweep should be repeated many times for a number of different HOM distributions representing manufacturing tolerances (typically 10). Putting that together, one would expect to use about 7 years of CPU time.

That seemed too long a time to wait. To make matters worse, the time to solve a single matrix goes roughly as $N_{\mathrm{HOM}}{ }^{3.6}$, so solving the case of for the CEBAF $12 \mathrm{GeV}$ upgrade with $800 \mathrm{HOMs}$ /axis rather than the FEL upgrade's 56 would require roughly 14,000 times the CPU time.

Fortunately, there are several ways to speed up the process. First, the eigenvalues' behavior is smoothly varying; an infinitesimal change in the frequency should only change an eigenvalue slightly. This means that an active search can be used; the frequency is changed up or down slightly and the eigenvalues tracked to determine the frequency at which they cross the positive real axis. The minimum frequency step size is then only limited by the numeric noise. The actual algorithm is somewhat more complicated by the presence of multiple eigenvalues and is discussed in detail in the tech note [7]. Previous versions of the code required the user to attempt to extrapolate the threshold from relatively widely scattered points; the iterative search removes those ambiguities.

Next, the size of the problem can be greatly reduced by considering only those HOMs whose frequencies are near to that in question and excluding those which have frequencies far away. The new version can divide the frequency range up into regions of interest (ROIs) and treat each as a separate problem.

Lastly, ignoring eigenvalues with components larger than a preset cutoff current simplifies the tracking. Typically, the cutoff current is set to be at least 10 times the threshold current of interest.
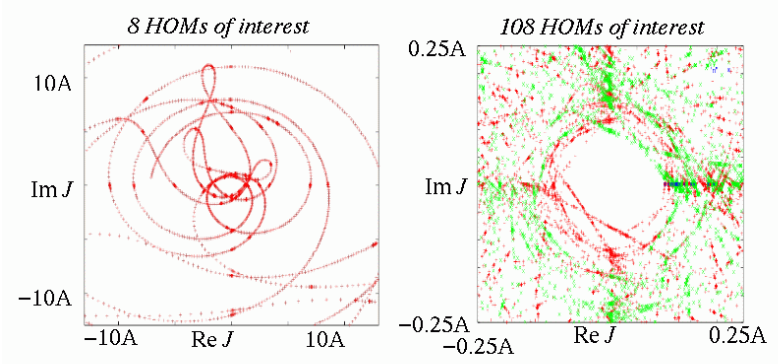

Figure 2. MATBBU output for 8 and $108 \mathrm{HOMs}$ of interest in the $10 \mathrm{~kW}$ FEL with a proposed supercell module.

In many cases of interest the exact frequency of each HOM in each cavity isn't known, but predicted to be distributed about a central value. It is then necessary to run multiple cases representing "as built" accelerators. MATBBU can now automatically generate and run input files with the appropriate distributions, and the results can then be shown as a scatter plot.

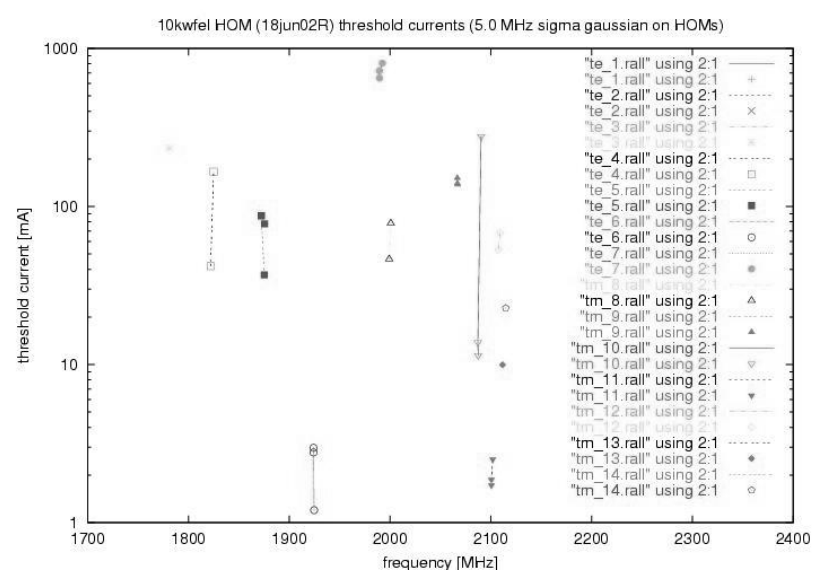

Figure 3. MATBBU predictions for 3 as-built FELs using a proposed module design and a $5 \mathrm{MHz}$ sigma spread in cavity HOM frequencies.

Generally, for modes with high Qs $\left(>10^{5}\right)$, the threshold current due to a given mode scales very nearly as $1 / \mathrm{Q}$. This property has been used to set the specification for the maximum Q for each HOM to avoid $\mathrm{BBU}$ at the design current. 


\section{COMPARISON}

Both TDBBU and MATBBU use the same input file and share much of their structure; both are written in FORTRAN77 with some ANSI C routines for portability and a command line interface. Both have been tested under a number of UNIX platforms, including GNULinux, HP-UX, SunOS, CrayOS, and IBM-AIX MATBBU may use either the IMSL ${ }^{[8]}$ or LAPACK $^{[9]}$ math library.

From a practical standpoint, the biggest difference in the codes is that TDBBU "sees" all the modes, while MATBBU only "sees" modes near near the frequency its scanning. Unless the number of HOMs is very large (1000s), for Qs $\sim 10^{6}$, MATBBU runs much faster than TDBBU (minutes vs. hours).

\begin{tabular}{|c|c|c|c|c|c|}
\hline Qrun & $\begin{array}{c}\text { Runtime } \\
{[\mathrm{uS}]}\end{array}$ & $\begin{array}{l}\text { Cray } \\
{[\mathrm{hr}]}\end{array}$ & $\begin{array}{c}\mathbf{X}[\mathbf{m A} \mathbf{A}] \\
\text { tdbbu } \\
(\mathbf{m a t b b u})\end{array}$ & $\begin{array}{c}\mathbf{Y}[\mathrm{mA}] \\
\text { tdbbu } \\
\text { (matbbu) }\end{array}$ & $\begin{array}{l}\text { Linux } \\
\text { [min] }\end{array}$ \\
\hline $1.0 \mathrm{E}+05$ & 150 & 0.09 & $128(120.7)$ & $120(147.2)$ & $\begin{array}{l}<1 \\
\text { min. }\end{array}$ \\
\hline $3.0 \mathrm{E}+05$ & 450 & 0.3 & $44(39.8)$ & 48 (48.7) & \\
\hline $1.0 \mathrm{E}+06$ & 1500 & 0.9 & 13 (11.9) & 15 (14.6) & \\
\hline $2.0 \mathrm{E}+06$ & 3000 & 1.8 & $(6.0)$ & (7.3) & \\
\hline $7.0 \mathrm{E}+06$ & 11400 & 7 & (1.7) & (2.1) & $\begin{array}{l}<1 \\
\text { min. }\end{array}$ \\
\hline
\end{tabular}

Table 1. Calculations using various $\mathrm{Q}_{\text {ext }}$ for a single mode with a $\mathrm{f}_{\mathrm{HOM}}=2114.8 \mathrm{MHz}$ and $\mathrm{R} / \mathrm{Q}=5.6 \mathrm{~W} / \mathrm{cm}^{2}$ in the central cavities of the $10 \mathrm{~kW}$ FEL.

To compare the codes, various test cases of for the 10 $\mathrm{kW}$ FEL were run and were found to agree to $\sim 10-20 \%$ when the only important HOMs were those within MATBBU's frequency range (Table 1).

\section{SUMMARY}

The MATBBU and TDBBU codes have been developed at Jlab and recently improved and used to predict the BBU thresholds for the CEBAF $12 \mathrm{GeV}$ upgrade and the $10 \mathrm{~kW}$ FEL upgrade. Both codes and their documentation will soon be available on the CASA ${ }^{[10]}$ web site.

\section{REFERENCES}

[1] G.A.Krafft, J.J. Bisognano and S.Laubach, Jlab Tech Note JLAB-TN-01-011

[2] J.J. Bisognano and R.L. Gluckstern, Proc. of 1988 Linear Accelerator Conf., 388 (1988)

[3] G.A. Krafft and J.J. Bisognano, 1987 PAC Proceedings, 1356 (1987)

[4] K.B. Beard, L. Merminga, B.Yunn, Jlab Tech. Note JLAB-TN-02-043

[5]K .B. Beard, L. Merminga, B.Yunn, Jlab Tech. Note JLAB-TN-02-045

[6] J.J. Bisognano and R.L. Gluckstern, 1987 PAC Proceedings, 1078 (1987)

[7] K .B. Beard, L. Merminga, B.Yunn, Jlab Tech. Note JLAB-TN-02-044

[8] Visual Numerics, Inc., http://www.vsi.com/products.imsl

[9] LAPACK's User Guide, E.Anderson et al, http://www.netlib.org/lapack/

[10] http://casa.jlab.org 